\title{
In-Situ Nanomechanics of Carbon Nanotubes and Graphene
}

\author{
Jian Yu Huang ${ }^{1}$, Feng Ding ${ }^{2}$, Qi Liang ${ }^{3}$, Ji Feng ${ }^{3}$, and $\mathrm{Ju} \mathrm{Li}^{3}$ \\ ${ }^{1}$ Center for Integrated Nanotechnologies (CINT), Sandia National Laboratories, Albuquerque, NM \\ 871855 \\ ${ }^{2}$ Institute of Textiles and Clothing, Hong Kong Polytechnic University, Kowloon, Hong Kong, P.R. China \\ ${ }^{3}$ Department of Materials Science and Engineering, University of Pennsylvania, Philadelphia, \\ Pennsylvania 19104
}

In this talk, I will review our recent progress in using a transmission electron microscopy-scanning probe microscopy (TEM-SPM) platform to probe the electrical and mechanical properties of carbon nanotubes and graphene [1]. First, individual multi-wall carbon nanotubes are peeled off layer-by-layer by electric breakdown inside the TEM. This provided new insights into the transport property of nanotubes. Second, plastic deformation, such as superplasticity, kink motion, dislocation climb, and vacancy migration, was discovered in nanotubes and graphene (see Fig. 1) for the first time. Third, we induced sublimation of suspended few-layer graphene by in-situ Joule-heating inside a TEM (see Fig. 2). The graphene sublimation fronts consisted of mostly $\{1100\}$ zigzag edges. Under appropriate conditions, fractal-like "coastline" morphology was observed. Extensive multiple-layer reconstructions at the graphene edges led to the formation of unique carbon nanostructures, such as $s p^{2}$-bonded bilayer edges (BLEs) and nanotubes connected to BLEs. Flat fullerenes/nanopods and nanotubes tunneling multiple layers of graphene sheets were also observed. Remarkably, more than $99 \%$ of the graphene edges observed during sublimation are BLEs rather than monolayer edges (MLEs), indicating that BLEs are the stable edges in graphene at high temperatures. We reproduced the "coastline" sublimation morphologies by kinetic Monte Carlo (kMC) simulations. The simulation revealed geometrical and topological features unique to quasi 2-dimensional (2D) graphene sublimation and reconstructions. These reconstructions were enabled by bending, which cannot occur in first-order phase transformations of $3 \mathrm{D}$ bulk materials. These results indicate that substrate of multiple-layer graphene can offer unique opportunities for tailoring carbon-based nanostructures and engineering novel nanodevices with complex topologies. Emerging directions of using the TEM-SPM platform to conduct cutting edge research in nanoscience and energy research areas will be highlighted.

\section{References}

1. J.Y. Huang et al., Nature 439, 281 (2006); J.Y. Huang et al., Phys. Rev. Lett. 94, 236802 (2005); 97, 075501 (2006); 98, 185501 (2007); 99, 175593 (2007); 100, 035503 (2008); J.Y. Huang et al., Phys. Rev. B, 78, 155436 (2008); PNAS 106, 10103 (2009); Nano Research 3, 43 (2010); J. Feng et al., Phys. Rev. B 80,165407 (2009)

This work was performed, in part, at the Center for Integrated Nanotechnologies, a U.S. Department of Energy, Office of Basic Energy Sciences user facility. Sandia National Laboratories is a multi-program laboratory operated by Sandia Corporation, a Lockheed-Martin Company, for the U. S. Department of Energy under Contract No. DE-AC04-94AL85000. 

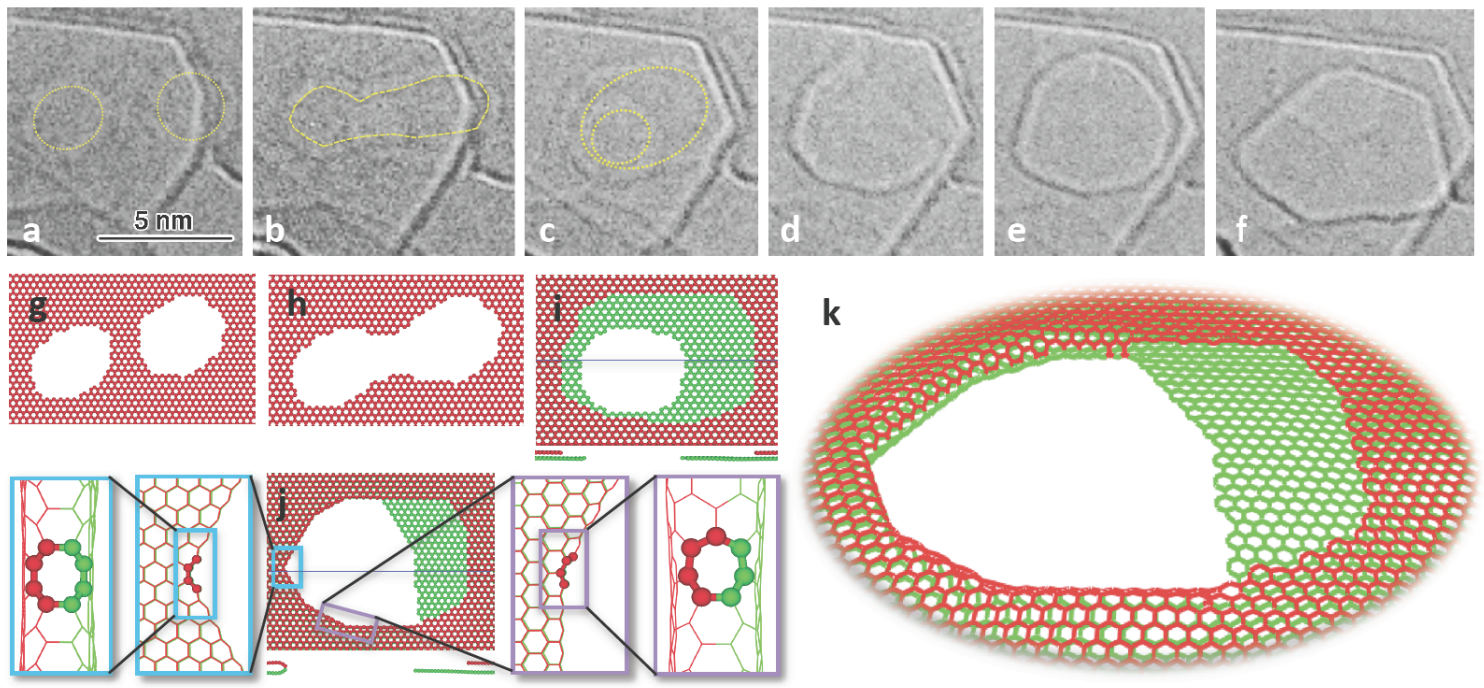

Fig. 1 The formation process of a bilayer edge vacancy hole (BLEVH) from merging of two monolayer edge vacancy holes (MLEVHs) in neighboring top and bottom graphene layers. Two MLEVHs appeared in (a) and (g) in the same graphene layer, and merged in (b) and (h) to form a bigger MLEVH. A new small MLEVH appeared in (c) and (i) (in the green layer), and it zipped with the big MLEVH in a neighboring red layer in (d), (j), and (k), forming a BLEVH. (e) The BLEVH quickly evolved into a polygonal shape (f). (k) A 3D view of zipping two MLEVHs to form a BLEVH.
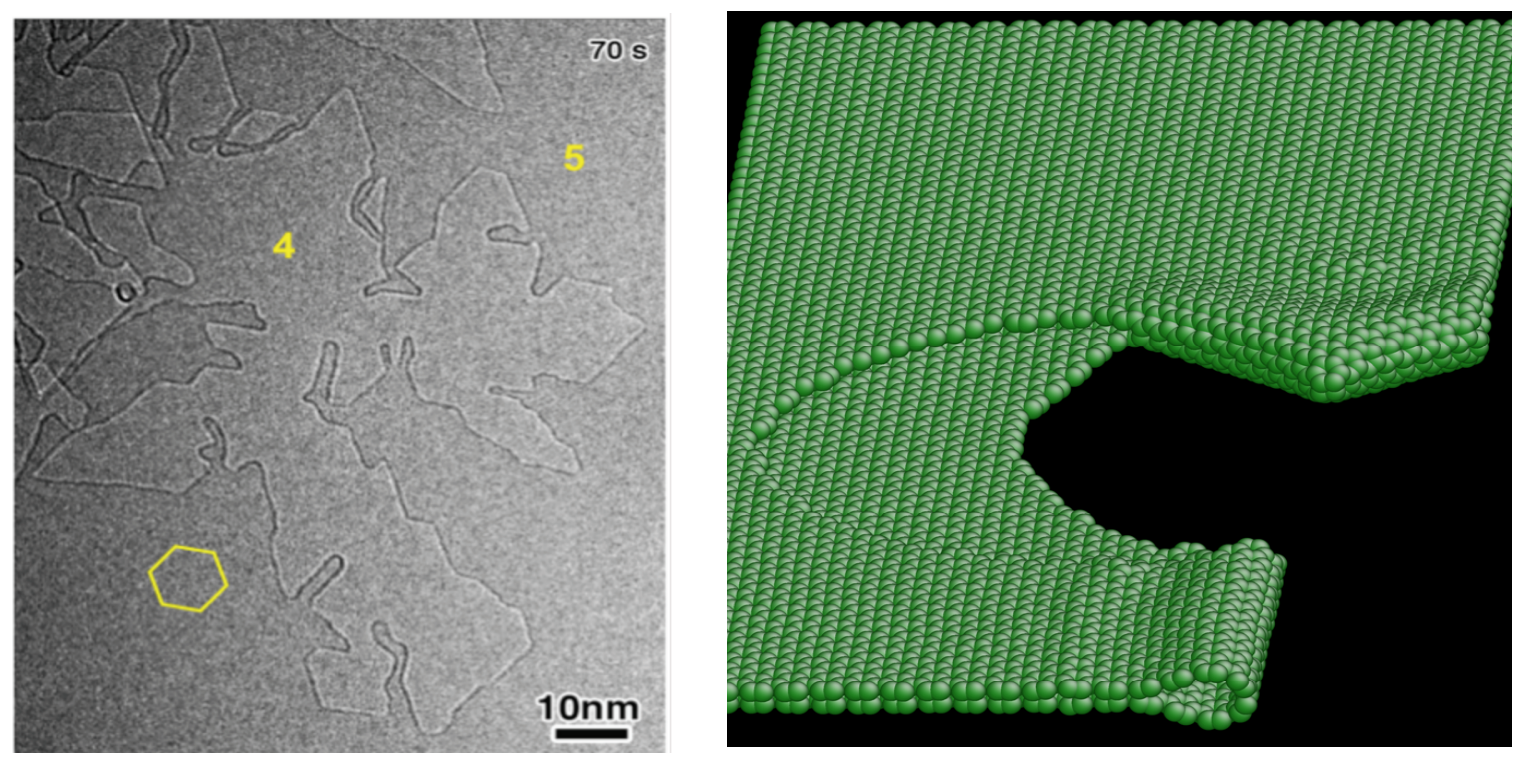

Fig. 2 (Left) A snow-flake-like graphene sublimation pattern formed by in-situ Joule heating. (Right) The graphene edges formed by sublimation are bilayer edges rather than monolayer edges. 